\title{
In vivo blockade of ovarian sympathetic activity by Neosaxitoxin prevents polycystic ovary in rats
}

\author{
Miguel del Campo1,2, Néstor Lagos ${ }^{1}$ and Hernán Lara² \\ 1'Laboratorio Bioquímica de Membrana, Departamento de Fisiología y Biofísica de la Facultad de Medicina de la Universidad de Chile, Santiago, Chile \\ ${ }^{2}$ Centre for Neurobiochemical Studies on Endocrine Diseases, Laboratory of Neurobiochemistry, Department of Biochemistry and Molecular Biology, \\ Faculty of Chemistry and Pharmaceutical Sciences, Universidad de Chile, Santiago de Chile, Chile
}

Correspondence should be addressed to H E Lara: hlara@ciq.uchile.cl

\begin{abstract}
A high sympathetic tone is observed in the development and maintenance of the polycystic ovary (PCO) phenotype in rats. Neosaxitoxin (NeoSTX) specifically blocks neuronal voltage-dependent $\mathrm{Na}+$ channels, and we studied the capacity of NeoSTX administered into the ovary to block sympathetic nerves and PCO phenotype that is induced by estradiol valerate (EV). The toxin was administered with a minipump inserted into the bursal cavity using two protocols: (1) the same day as EV administration and (2) 30 days after $E V$ to block the final step of cyst development and maintenance of the condition. We studied the estrous cycling activity, follicular morphology, steroid plasma levels, and norepinephrine concentration. NeoSTX administered together with EV decreased NA intraovarian levels that were induced by EV, increased the number of corpora lutea, decreased the number of follicular cyst found after EV administration, and decreased the previously increased testosterone plasma levels induced by the PCO phenotype. Estrous cycling activity also recovered. NeoSTX applied after 30 days of EV administration showed near recovery of ovary function, suggesting that there is a specific window in which follicular development could be protected from cystic development. In addition, plasma testosterone levels decreased while those of progesterone increased. Our data strongly suggest that chronic inhibition of sympathetic nerves by a locally applied long-lasting toxin is a new tool to manage the polycystic phenotype in the rat and could be applied to other mammals depending on sympathetic nerve activity.
\end{abstract}

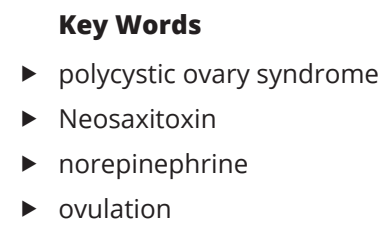

Journal of Endocrinology (2020) 244, 523-533

\section{Introduction}

High testosterone plasma levels and the presence of fluidfilled follicles (cysts) that do not ovulate characterize polycystic ovary syndrome (PCOS). PCOS is the most prevalent ovarian pathology in women, affecting $5-12 \%$ of women of reproductive age, and it is characterized by many endocrine/metabolic associated pathologies.
The most important component of these disorders is the anovulation, which results in irregular menstruation and infertility. In addition, PCOS patients are associated with having masculinizing effects, which result from the hyperandrogenism. It is known that the aberrant polycystic morphology can be restored to normal 
follicular development by the following: (1) suppression of sympathetic activity resulting from surgical resection of the superior ovarian nerve (SON; the principal sympathetic nerve controlling follicular development and steroid secretion (Lara et al. 1990a, 1993, Flores et al. 2011, Del Campo et al. 2019); (2) administration of the $\beta$-adrenergic antagonist (Luna et al. 2012); and (3) blockade of the nerve activity with a locally implanted microstimulator (Pikov et al. 2018). For humans, acupuncture (to block the ovarian sympathetic nerves) was found to reduce the PCOS symptoms (Stener-Victorin et al. 2008). Current PCOS treatments include estrogen receptor modulators, gonadotropins, laparoscopic ovarian drilling, weight loss, and exercise (Banaszewska et al. 2019). However, these treatments are focused on the symptoms rather than the underlying cause and are moderately effective.

The current PCOS therapies do not represent the brainovary connection in the human body. While ovulation is controlled by pituitary-secreted gonadotropins (luteinizing hormone and follicle-stimulating hormone), it is also strongly influenced by the sympathetic neural control (Lara et al. 2002). The mammalian ovary receives well-organized innervation of postganglionic sympathetic nerves, which originates from the ovarian ganglion and from the celiac and renal plexi (Burden et al. 1985, Curry et al. 1985). Sympathetic innervation reaches the ovary via the following two nerves: (1) the ovarian plexus nerve (OPN), which is associated with the ovarian branch of the uterine artery (Neilson et al. 1970); and (2) the SON, which is at least partially associated with the ligament of the ovary (Neilson et al. 1970, Lawrence \& Burden 1980). The SON fibers predominantly innervate the secretory components of the ovary, i.e. interstitial glands and follicles, whereas the OPN fibers are mostly perivascular (Lawrence \& Burden 1980). Although there is some variation in the way that sympathetic nerves reach the ovary, no differences have been found in the intraovarian distribution of sympathetic fibers, which is similar in all mammalian species, although the density of the network varies considerably among them (Jacobowitz \& Wallach 1967). Norepinephrine is the main neurotransmitter that is present in the ovary (Lara et al. 1990b, 2002, Greiner et al. 2005). The innervation of the gland has been shown to be involved in the regulation of ovarian-specific functions, such as steroidogenesis and early follicular development (Lara et al. 2002, Greiner et al. 2005) by activating $\beta$-adrenergic receptors that are present in cells of the ovarian follicle.

Transection of the SON, which carries the bulk of the sympathetic innervation to the ovarian endocrine cells, has been observed to restore estrous cyclicity and ovulation (Barria et al. 1993). However, a sustained increase in sympathetic activity by estradiol administration (Lara et al. 2000), chronic sympathetic stress (Dorfman et al. 2003), or pharmacological $\beta$-adrenergic receptor activation (Luna et al. 2012) causes a polycystic phenotype in the rat ovary, which, in many aspects, resembles PCOS in women.

In this study, we evaluated the effect of suppressing the sympathetic nerve activity in the ovary by local chronic administration of Neosaxitoxin (NeoSTX), a toxin that specifically blocks the voltage-dependent $\mathrm{Na}+$ channels with a high affinity and long half-life (Llewellyn 2006, Alonso et al. 2016). NeoSTX was administered using a mini-osmotic pump for 30 days as a potential treatment for PCOS. NeoSTX has been previously shown to produce an effective and reversible block of nerve conduction in many sensorial and autonomic nerves and its successful use as a therapeutic agent to treat multiple pathologies in human and veterinary medicine is extensive and well documented (Rodriguez-Navarro et al. 2007a,b, Lobo et al. 2015, Manriquez et al. 2015, Riquelme et al. 2018).

\section{Materials and methods}

\section{Study design}

All animal studies were performed in accordance with the animal protocols that were approved by the Bioethics Committee of the Faculty of Chemistry and Pharmaceutical Sciences at the Universidad de Chile (UCh), Code CBE2017-05. They also complied with national guidelines (CONICYT Guide for the Care and Use of Laboratory Animals).

\section{NeoSTX purification}

NeoSTX was isolated and purified in the Membrane Laboratory of the Physiology and Biophysics Department, Faculty of Medicine, Universidad de Chile. NeoSTX is produced by dinoflagellates from the genus Alexandrium, and it was purified from shellfish that have accumulated this drug and were collected by our laboratory in the southern macrozone of Chile (Manriquez et al. 2015). The structure and purity was tested using high-pressure liquid chromatography with a post column derivatization, as previously described (Zamorano et al. 2013, Garcia et al. 2015). This preparation has been used in many clinical studies as a local anesthetic over the last 10 years. 


\section{Animals}

We used Sprague-Dawley female rats from our facilities. The rats were maintained under a 12-h:12-h dark:light cycle at $21 \pm 1^{\circ} \mathrm{C}$ during all the experiments. They were fed Labdiet rat food (Brentwood, MO, USA), provided with enrichment, and co-housed with two other rats of the same age to maintain normal behavior. In control experiments, 3-month-old rats were hemi-ovariectomized (ULO) during the minipump implantation process or sham operation to eliminate a putative effect of the contralateral ovary on steroid secretion that could modify the hypothalamic activity. Some asymmetry was described between the right and left ovary regarding the hypothalamic control of the ovary (Pastelin et al. 2017, Rosas et al. 2018). Each ovary has a different capacity to release steroids; thus, a hormonal signal from one ovary could affect the contralateral ovary by altering the hormonal signals that arrive at the ovary via the circulation. This was the reason that all control (either sham-injected or sham-denervated) rats underwent ULO to compare the same conditions in all the groups. In the experiments with the PCOS phenotype (Group 3), rats (24 days old) were injected with estradiol valerate $(\mathrm{EV})$ in a single i.m. dose of $10 \mathrm{mg} / \mathrm{kg}$ of $\mathrm{EV}$ (Sigma Chemical Co.) dissolved in sesame oil. This age group was selected because we previously demonstrated (Cruz et al. 2012) a developmental window in which the administration of a single EV solution injection produced a permanent effect on the ovary. Twenty-four days of age was selected as the age limit for reversion, and the PCO phenotype was established when the rats were killed. Control rats were injected with sesame oil. To eliminate the hypertrophy that was derived in the contralateral ovary after ULO (Pastelin et al. 2017, Rosas et al. 2018), we used both ovaries in this experiment. Thus, we implanted one minipump in each ovary.

The rats were randomly assigned to the following groups, as described below.

\section{Group 1: Effect of NeoSTX on the ovarian noradrenaline (NA) after transmural stimulation in vitro}

Rats were divided into NeoSTX and Sham groups. Six adults rats in estrous were killed and the two ovaries were incubated in Krebs Ringer Phosphate buffer gassed with $95 \% \mathrm{O}_{2}$ and $5 \% \mathrm{CO}_{2}$ and incubated at $37^{\circ} \mathrm{C}$ for $10 \mathrm{~min}$ in the presence of $10 \mathrm{mM}$ Tetraethylammonium (TEA), which causes profound facilitation of neurotransmitter release and a significant reduction in tissue NA content when the organ is electrically stimulated (Wakade 1979, 1980, Lara et al. 1985). The left ovary was incubated with $10 \mathrm{nM}$ NeoSTX, and the right ovary was incubated without NeoSTX.

The ovaries were placed in a perfusion chamber for transmural stimulation using a SD5 Grass stimulator (Grass Instruments, Quincy, Mass, USA), with trains of 150 pulses $(80 \mathrm{~V}, 1 \mathrm{~ms}$ duration), at $5 \mathrm{~Hz}$ every $30 \mathrm{~s}$ for $90 \mathrm{~min}$, separately, with infusion $(2 \mathrm{~mL} / \mathrm{min})$ of the respective buffers. At the end of the stimulation period, the ovaries were harvested for NA determination.

In the basal group, four adults rats in estrous were euthanized and the two ovaries were incubated in TEA-Krebs-ringer-phosphate buffer under the same experimental conditions as the experimental groups for 90 min, separately, before being stored.

\section{Group 2: Chronic effect of NeoSTX on the ovary of adult control rats}

Two experimental series were performed. In the first test, ten adult rats were randomized and separated into two groups of five rats (sham and NeoSTX groups). During the surgical process, the right ovary was removed and frozen at $-80^{\circ} \mathrm{C}$ for noradrenaline (NA) determination. The left ovary from the rats in the NeoSTX group was implanted with a osmotic mini pump (Alzet osmotic pump, Model 2004, Durect Corporation Cupertino, CA, USA) filled with a solution of NeoSTX dissolved in saline. The pump delivered $0.2 \mathrm{nmol} /$ day of the toxin for 28 days. The mini pump was inserted with a cannula that was placed directly onto the bursal cavity, cemented with surgical glue (Histoacryl blue, B Braun Surgical, Rubi, Spain), and maintained for 28 days. Sham-operated rats were subjected to the same procedure, but a cannula was inserted in the bursal cavity with no drug. Rats were also killed on day 30 after implantation. The left ovary was used for NA determination.

In the second group, 14 adults rats were randomized and separated into two groups of seven rats each (sham and NeoSTX groups). The same surgical procedure was followed as for the first experimental series, except that plasma from all rats was collected and saved for hormone determination when the rats were killed. The left ovary was halved, and one half was fixed in Bouin's fixative for morphological analysis and the other half was used for NA determination. One of the ovaries per group was lost in the sample processing for morphological analysis. https://joe.bioscientifica.com

https://doi.org/10.1530/JOE-19-0545 (c) 2020 Society for Endocrinology Published by Bioscientifica Ltd.
Printed in Great Britain 


\section{Group 3: The effect of NeoSTX on the PCO-induced phenotype by estradiol valerate}

The objective of this experiment was to determine if blocking NA activity can prevent the development of the PCO phenotype or reverse PCOS that was previously induced by the EV administration in rats. Because of a possible reversion of the EV effect over time after EV (90 days), the rats used in this experiment were injected when they were 24 days old. We have shown that rats injected with EV at this age had no reversion of the PCO phenotype (Cruz et al. 2012). Control rats were injected with sesame oil and the EV-treated rats received a single dose of $10 \mathrm{mg} / \mathrm{kg}$ of EV (Sigma Chemical Co.) dissolved in sesame oil.

For the study of PCO phenotype prevention, 24-dayold rats were injected with EV. On the same day, an osmotic pump containing NeoSTX was implanted in both ovaries and it delivered the toxin for 28 days. Rats were killed 60 days thereafter.

For the study of PCO phenotype reversion, 24-day-old rats were injected with $\mathrm{EV}$, and after 30 days (rats were 54 days old), an osmotic minipump with NeoSTX was implanted in both ovaries.

These experimental groups were compared with rats that were injected with EV at 24 days old and rats that were injected with sesame oil. In addition, in rats injected only with the vehicle, the mini pump with NeoSTX was placed on day 54 in both ovaries (Table 1). Plasma was collected and the left ovary was preserved in Bouin's fixative to evaluate the morphology and the right ovary was used for NA determination.

Our primary outcome measures were evaluated in the animals from the following experimental groups: Estrous cycle phase, evaluated daily by vaginal cytology; Ovarian morphological phenotype; Ovarian NA concentration; and Steroid plasma levels.

\section{Estrous cycling activity}

Estrous cycling activity was assessed daily beginning at puberty to the end of the procedure, and this included a fertility test. Vaginal fluid was obtained daily using a plastic pipette and it was placed on the glass slide and observed under a light microscope. Epithelial cells (large, round, nucleated), cornified cells (irregular, without nucleus), and leukocytes (small, round) were identified, and their proportion was analyzed to define proestrus during the night of ovulation (Dissen et al. 1996), estrus (the day after ovulation), and diestrus (two previous days before P), as previously described (Marcondes et al. 2002).

\section{Analysis of follicular structures}

One of the halves or the entire ovary was fixed in Bouin's fixative, cut into $6-\mu \mathrm{m}$ slices, stained in hematoxylineosin, and analyzed. The presence of precystic structures or follicular cyst and corpora lutea was quantified. These structures were dominant in the area that was covered in all the ovaries that were analyzed. The following criteria were defined to quantify follicular structures: cystic follicles were devoid of oocytes and showed a large antral cavity; a well-defined thecal cell layer; and a thin (mostly monolayer) granulosa cell compartment containing apparently healthy cells. Type III follicles have oocytes that are not completely surrounded by granulosa cells, which have discontinuous and uneven layers, with a predominant antrum. The corpora lutea showed the absence of oocytes and the presence of luteal cells instead of granulosa cells (Lara et al. 2000).

\section{Quantification of intraovarian NE levels}

Quantification of NE levels was performed using the competitive norepinephrine ELISA kit-Research ${ }^{\circledR}$ (IMMUSMOL, Pessac, France), according to the manufacturer's instructions. NE was extracted using a cisdiol-specific affinity gel, acylated, and then derivatized enzymatically. The antigen was bound to the solid phase of the microtiter plate. The derivatized standards, controls, and samples, as well as the solid-phase-bound analyte, competed for a fixed number of antiserumbinding sites. The antibody bound to the solid phase was detected using an anti-rabbit IgG-peroxidase conjugate

Table 1 Experimental groups to study the reversion or prevention of the EV-induced PCO phenotype by in vivo local delivery of NeoSTX using mini-osmotic pumps.

\begin{tabular}{lll} 
& \multicolumn{1}{c}{ Age } \\
& Group & Sham \\
5 & NeoSTX \\
5 & EV \\
5 & EV + NeoSTX (24 d) \\
5 & EV + NeoSTX (54 d) \\
\hline
\end{tabular}

https://joe.bioscientifica.com https://doi.org/10.1530/JOE-19-0545

\begin{tabular}{l}
\hline \multicolumn{1}{c}{$\mathbf{2 4}$ days old } \\
\hline Drug administration \\
\hline Sesame oil injected \\
Sesame oil injected \\
EV \\
EV + NeoSTX \\
EV
\end{tabular}

\begin{tabular}{l} 
54 days old \\
\hline Drug administration \\
\hline- \\
NeoSTX \\
- \\
- \\
NeoSTX
\end{tabular}

\begin{tabular}{l}
\hline \multicolumn{1}{c}{$\mathbf{8 4}$ days old } \\
\hline Euthanasia \\
\hline EU \\
EU \\
EU \\
EU \\
EU
\end{tabular}

(c) 2020 Society for Endocrinology Published by Bioscientifica Ltd. Printed in Great Britain 
and tetramethylbenzidine (TMB) as a substrate. The reaction is monitored at $450 \mathrm{~nm}$. The sensitivity was $2 \mathrm{pg} / \mathrm{mL}$, and the intra and interassay variability were 8.4 and $8.0 \%$, respectively. The cross-reactivity found was $0.14 \%$ for adrenaline and $1.8 \%$ for dopamine.

\section{Plasma levels of steroid hormones}

Plasma levels of steroid hormones testosterone and progesterone were measured. Serum testosterone and progesterone levels were determined using an EIA, according to the manufacturer's instructions. The catalog number of the test kits were 11-TESHU-E01 and 11-PROHU-E01 for $\mathrm{T}$ and P4, respectively (Alpco Diagnostic, Windham, NH, USA). Intra- and interassay variations were less than $6 \%$ for $\mathrm{T}$, and less than $5 \%$ for $\mathrm{P} 4$. The minimal detectable values were $0.02 \mathrm{ng} / \mathrm{mL}$, and $0.1 \mathrm{ng} / \mathrm{mL}$, respectively.

\section{Statistical analysis}

Differences between the control and treatment groups were analyzed using a paired $t$-test or unpaired $t$-test, according to the comparison group. For within-animal comparisons, the two-tailed $t$-test was used. Significance was set at $P<0.05$. The minimum number of rats that were used for the experiments was calculated as described by Zar (Zar 1984). The Shapiro-Wilk test was applied to verify the normal distribution of the data. All statistical analyses were performed using GraphPad Prism v5.0 software (GraphPad Software).

\section{Results}

\section{The effect of NeoSTX on ovarian noradrenaline levels}

To determine if NeoSTX blocks ovarian sympathetic activity, we designed an in vitro assay in which we incubated rat ovary to apply a transmural train of 150 pulses at $5 \mathrm{~Hz}$ every $30 \mathrm{~s}$ of $1 \mathrm{~min}$ for $90 \mathrm{~min}$ (80 V; duration, $1 \mathrm{~ms})$. The acute stimulation in the presence of TEA (a blocker of the K-repolarizing current) was used by Wakade (Wakade 1980) to effectively decrease the NA content in the vas deferens. Stimulation for 90 min decreased the NA content in the ovary by $50 \%$ (Fig. 1A). When the same experiment was performed in the presence of NeoSTX $(100 \mathrm{nM})$, there was no decrease in NA content and a small increase in the neurotransmitter (Fig. 1A).

Figure 1B shows the results that were obtained after intraovarian chronic administration of NeoSTX in vivo

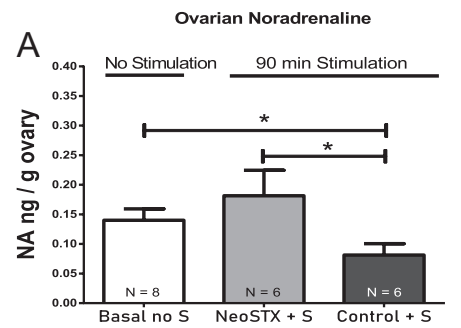

B
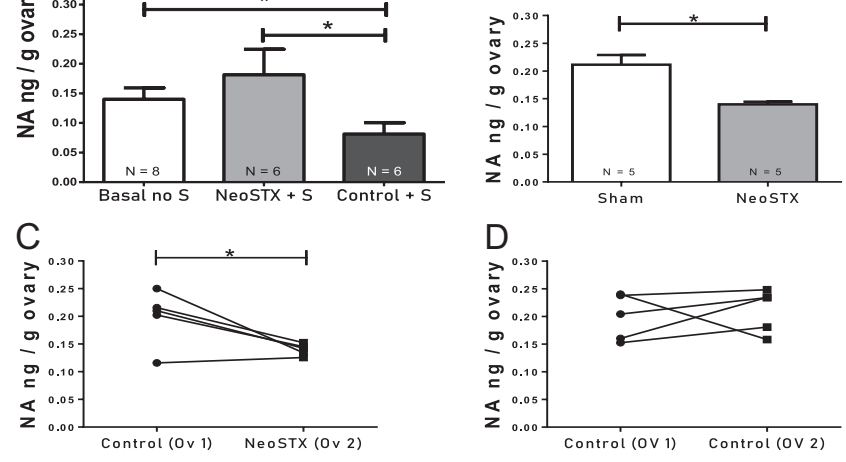

\section{Figure 1}

The effect of NeoSTX on ovarian norepinephrine in vitro and in vivo. (A) Norepinephrine content in ovaries exposed to transmural stimulation (S), with or without NeoSTX in TEA-Krebs buffer, for $90 \mathrm{~min}$. The basal level corresponds to incubation of ovaries without stimulation. (B, C and D) Norepinephrine content in ovaries that received chronic (28 days) administration of NeoSTX (0.1 $\mu \mathrm{g} /$ day) compared to the sham group; (B) compared to the contralateral ovary that was removed on day 0 (Ov 1) with that removed on day 28 (Ov2); and (C) compared to both ovaries of the sham group (D). * $P<0.05, t$-test.

using an osmotic minipump for 28 days. Rats underwent ULO at time 0 and the ovary was used as a 0 time control either for sham or NeoSTX rats. Ovarian noradrenaline decreased by $30 \%$ by the presence of NeoSTX (Fig. 1B). In Fig. 1C and D, the individual response of the ovaries after exposure or no exposure to NeoSTX for 28 days.

\section{The effect of NeoSTX on the changes induced by EV on the ovarian corpora lutea, cyst, and plasma levels of steroid hormones}

NeoSTX administered to control adults rats also modified the follicular development. There was an increase in the number of corpora lutea and a decrease in the number of follicular cysts that were normally seen in the control ovaries (Fig. 2A and B). Plasma levels of progesterone were also increased in the ovaries of rats treated with NeoSTX (Fig. 2C). Although NeoSTX reduced the number of cysts, there was no decrease in plasma testosterone levels (Fig. 2D).

\section{The effect of NeoSTX on the development and maintenance of the polycystic phenotype}

This experiment was designed to analyze if NeoSTX could prevent $(\mathrm{P})$ or reverse $(\mathrm{R})$ the PCO phenotype that was induced by EV. In this case, we used five groups: (1) Sham; (2) NeoSTX; (3) EV; (4) EV+NeoSTX (to prevent); 
A

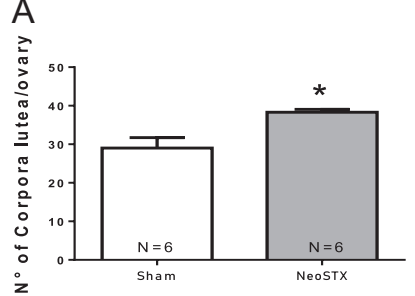

B
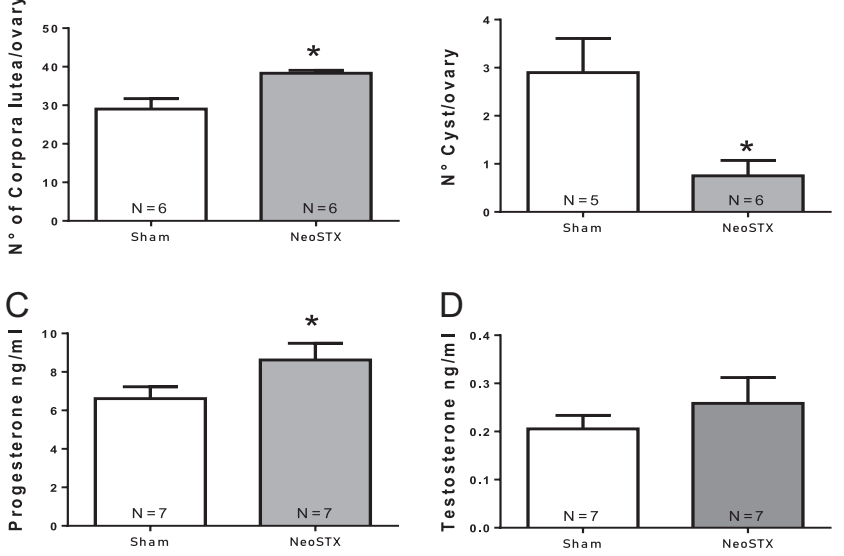

Figure 2

Effect of chronic NeoSTX administration over the follicular development and steroid plasma levels in adult control rats. (A and B) Quantification of the follicular morphology of luteal bodies (A) and cysts (B). (C and D) Progesterone (C) and testosterone (D) plasma levels. ** $P<0.01, t$-test, $\star p<0.05$.

and (5) EV + NeoSTX after 28 days (to reverse). EV or vehicle were administered at 24 days old and the experiment was complete after 60 days (Table 1 ).

A representative image of the ovaries from EV-treated rats is shown in Fig. 3. In the ovaries of the EV group, there were almost no corpora lutea, but the ovary was filled with follicular cysts. Under conditions in which EV and NeoSTX were used, the most prevalent structure was the corpora lutea.

Figure 4A shows the estrous cycling activity of the EV-injected rats. All rats had an open vagina (a signal of puberty in the rat) between 24 and 26 days old. EV rats did not show a regular estrous cycle (transition from diestrus to proestrus, estrus, and metestrus). In the rats that received NeoSTX administration at 24 days old (when EV was administered), the estrous cycling activity was reversed to an almost regular profile for rats that showed 4-5 days for the length of each cycle during all observation periods. NeoSTX administration
28 days after EV administration (i.e. when the polycystic condition is almost developed) did not restore estrus cycling activity up to when the toxin was applied at 28 days, after which there was a reversion of the acyclic condition to a regular profile. Quantification of the number of regular estrous cycles that occurred during the experimental period is shown in Fig. 4B. After NeoSTX administration, either at the beginning or after 28 days of EV administration, there was a recovery of the estrous cycling activity.

Corpora lutea quantification was also consistent with the ovary image (Fig. 4C). The simultaneous administration of NeoSTX to the EV-treated rats resulted in a greater number of corpora lutea compared to the rats treated with EV only. The same effect was found when NeoSTX was applied 30 days after EV. Follicular cysts decreased when the NeoSTX was simultaneously administered with EV (Fig. 4D). The same result but at a lower magnitude was found when the NeoSTX was administered 28 days after the EV-induced cyst formation, which supports that in this case, the ovary function is oriented toward ovulation and not toward PCO development. Rats treated with NeoSTX had a follicular morphology where the corpora lutea predominated, and it was similar or greater in number than the sham control.

Prevention of PCO by NeoSTX (EV+simultaneous NeoSTX) was accompanied by a significant decrease in the ovarian norepinephrine concentration (Fig. 5A). There was no significant decrease in ovarian norepinephrine when NeoSTX was administered after 30 days of EV administration. Similarly, the simultaneous administration of EV and NeoSTX increased the plasma levels of progesterone (Fig. 5B). A similar situation was found with testosterone plasma levels (Fig. 5C). Testosterone plasma levels decreased when NeoSTX was administered on the same day as EV, but it did not recover when the toxin was administered 30 days after EV (Fig. 5C). Figure 6 shows a proposed model to explain the principal findings of this work.
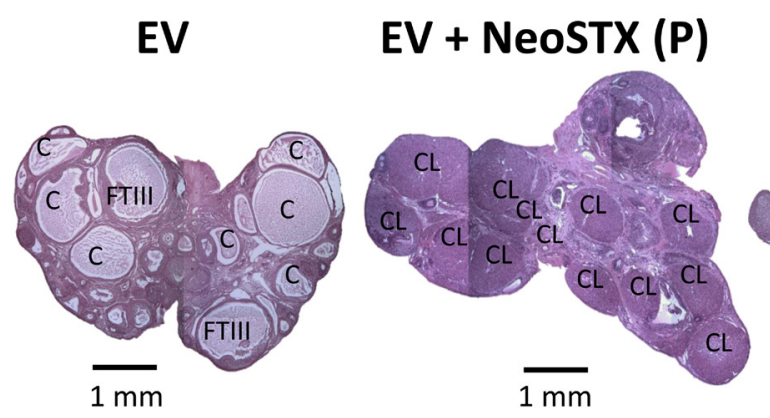

\section{$E V+\operatorname{NeoSTX}(R)$}

\section{Figure 3}

(C) Morphological appearance of the ovaries treated with NeoSTX to prevent $(\mathrm{P})$ or reverse (R) the cystic phenotype generated by EV. CL, corpus luteum; C, cyst; FTIII, follicle type III. A

representative image per group is shown. A full colour version of this figure is available at https:// doi.org/10.1530/JOE-19-545. 
A EV

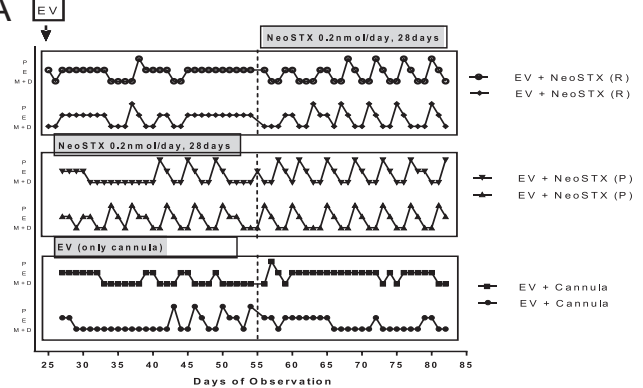

B

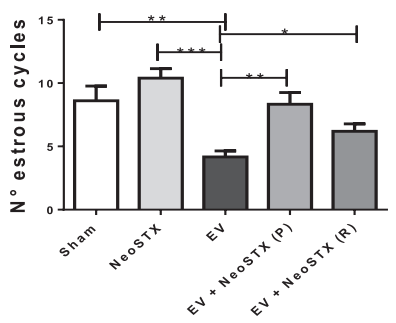

C

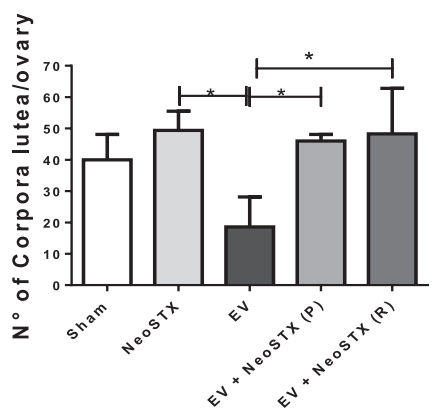

\section{Discussion}

In this research, we studied the effect of NeoSTX, a toxin that naturally accumulates in shellfish (Manriquez et al. 2015). NeoSTX presents a high affinity for neuronal $\mathrm{Na}$ channels that are involved in neuronal depolarization of the rat ovary in the control and PCO phenotype that was induced by EV administration. The NeoSTX acts as a specific
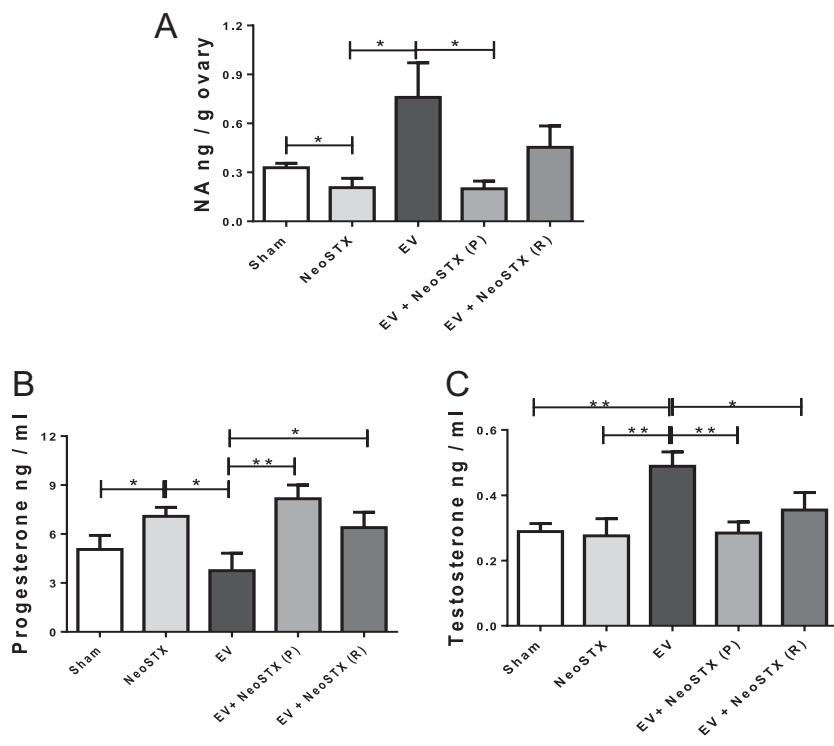

Figure 5

Effect of chronic NeoSTX administration in the concentration of intraovarian NA (A) and on progesterone (B) and testosterone (C) plasma levels when NeoSTX was administered to prevent $(P)$ or to reverse $(R)$ the cystic phenotype generated by EV. ${ }^{*} P<0.01 ;{ }^{\star} P<0.05$, $t$-test.

Figure 6 https://joe.bioscientifica.com https://doi.org/10.1530/JOE-19-0545
(C) 2020 Society for Endocrinology Published by Bioscientifica Ltd. Printed in Great Britain

\section{Figure 4}

Effect of chronic NeoSTX administration on the prevention $(P)$ and reversal $(R)$ of the cystic phenotype generated by EV. $(A)$ is a representative profile of the estrous cyclic. Estrous cycles were analyzed in 25-day-old rats (from the vaginal opening). EV was administered to all groups. The upper representative pair of rats in the upper part of the $(A)$ correspond to NeoSTX administered to group R (R: reversion) on day 28 after EV and the minipump remained patent for 28 more days. The middle pair of estrous cycles corresponds to NeoSTX that is applied to the group P (P: prevention). NeoSTX was administered together with EV and minipump remained patent for 28 days. On day 84 , rats were euthanized (EU). The pair of rats at the bottom represent the sham-operated rats that only received the initial EV administration on day 24 (sham without cannula). Two representative panels are shown per group. $\mathrm{P}$, Proestro; E, Estro; $M+D$, Metaestro and Diestro. (B) Quantification of the number of estrous cycles in 4 or 5 days per group. (C) Quantification of the number of corpora lutea. (D) Quantification of the number of cysts. ${ }^{*} P<0.01 ; * P<0.05, t$-test.

inhibitor of the neurotransmitter release. In the first part of the work, we tested the capacity of the toxin to regulate the sympathetic nerve terminal activity in vitro and by chronic exposure in vivo. Transmural electric stimulation with supramaximal stimulation led to a decrease in the norepinephrine content by increasing neurotransmitter release. We did experiments in the presence of TEA to block the $\mathrm{K}^{+}$-repolarizing channel and, thus, maintain the nerve terminals in the depolarized state so they continued to release more neurotransmitter (Wakade 1979, 1980,

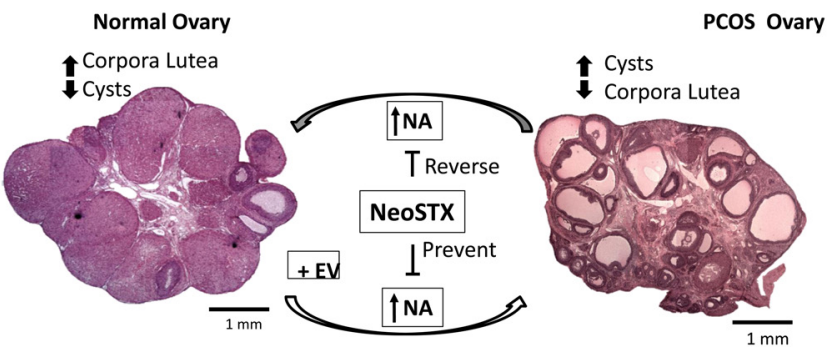

Proposed model: NeoSTX blocks the voltage dependent $\mathrm{Na}+$ channel in ovarian neurons and sympathetic nerve activity, and a.- prevent the development of PCO phenotype when NA increases as a consequence of EV administration. The second process is when the NeoSTX is applied at the time of development of the first cohort of cystic follicles (30 days after $\mathrm{EV}$ ), it was able to block cyst production and, thus, reverse the PCO phenotype. The data are supported by the in vivo decrease of intraovarian $\mathrm{NA}$, the increase in corpora lutea, and decrease in the cystic follicles that were previously increased by EV. It also increases progesterone and decreases plasma testosterone, which strongly suggests the recovery of follicular steroid synthesis. A full colour version of this figure is available at https://doi.org/10.1530/JOE-19-545. 
Lara et al. 1985). Using this system, we can rapidly test the size of the releasable pool of neurotransmitter and, thus, the activity of the nerves. Ninety minutes of stimulation in this condition decreased the NA content by $50 \%$, which represents a supramaximal stimulation, indicated that it was a good model in which to study the effect of NeoSTX as a blocker of the $\mathrm{Na}^{+}$channel to eliminate the action potential generated by depolarization in the tissue. The finding that NeoSTX blocked the decrease in ovarian NA induced by stimulation means that the toxin was effective at blocking ovarian nerve activity. Because we found a similar decrease in NA after the chronic implantation of a minipump with the toxin for 28 days, this suggests that there is a decrease of the sympathetic tone in the ovary in a chronic fashion that covers the entire development of a ovarian follicle cohort. This also suggests that study of the effect on ovarian steroids secretion is required.

The increase in the number of corpora lutea and the decrease in follicular cyst found after 30 days of chronic NeoSTX exposure suggests that that, under normal conditions, the sympathetic activity in the ovary maintains a basal tone that is necessary to control follicular development and in steroid secretion. Similar results to those obtained after surgical denervation of the hyper-noradrenergic state after EV administration (Lara et al. 1993). This effect was similar to the changes in follicular development that we recently described when we increased the cholinergic tone to the ovary of normal rats keeping norepinephrine constant (Urra et al. 2016, Riquelme et al. 2019). It is likely that the ovary function is mainly controlled by the hypothalamicpituitary axis and the role of the autonomic tone results from a balance between cholinergic and noradrenergic activity. For example, a change in this balance to a hypernoradrenergic state turns the ovary into a polycystic ovary, while a change to a cholinergic predominance is the healthy condition (Riquelme et al. 2019). The concept that seems new for a typically endocrine organ is commonly found in all organs receiving autonomic control such as heart or the renal system (Velasquez et al. 2019, Grisk 2020). Independent of the impact on ovarian function, this strengthens the concept that endocrine pathologies can be managed by modifying the autonomic tone to the organ (Lansdown \& Rees 2012).

Recently, neural regulation was developed as a new therapeutic tool to treat different pathologies (Birmingham et al. 2014, Chavan \& Tracey 2017, Pavlov \& Tracey 2017, Velasquez et al. 2019, Grisk 2020). The ovary is one of the organs in which sympathetic activity has been studied (Lara et al. 2002, Lansdown \& Rees 2012).
This situation has been explored especially because of the close relationship between stress and reproductive function (Toufexis et al. 2014). Many studies that were done in rodents have described the organization of sympathetic innervation of the ovary, their activity, and the relationship of the nerves to specific functions of the ovary such as steroid secretion and follicular development. Although there is strong evidence of a hyper-noradrenergic tone as a part of the causative factors in the development of the PCO phenotype in rodents (Barria et al. 1993), there are few studies on the role of sympathetic nerves in the PCOS in humans (Stener-Victorin et al. 2008, 2009, Zheng et al. 2015). These authors published some studies where acupuncture methods were used, which can lower the sympathetic nerve activity and increase the rate of ovulation in women with PCOS. The most recent studies, however, have described that this technique is not as effective as what was previously described (Kuang et al. 2018, Chen \& Lim 2019, Lim et al. 2019). Most promising are recent studies in rodents in which electronic devices were implanted in the SON to regulate the nerve rate of discharge and thereby regulate the neuronal input to the ovary (Pikov et al. 2018).

$\beta$-Adrenergic blockers such as propranolol have been used to block the PCO phenotype induced by $\beta$-adrenergic stimulation induced by isoproterenol in rats (Luna et al. 2012). They have the problem of a short half-life (4-5 h, which requires repeated doses as we did in the study), and because of the systemic administration, many tissues are targeted for its action. Interestingly recently appeared a paper in which an acute dose of locally applied propranolol facilitates ovulation (Venegas et al. 2019). Although the delivery system could be improved using targeted nanodelivery systems, the short half-life of the drug in solution limits the development of new products. The advantage of using NeoSTX is the very good affinity for the $\mathrm{Na}^{+}$channel and its long-lasting effect. Thus, it could be a good alternative to block neuronal systems for longer times and, therefore, be useful in various pathologies to decrease the sympathetic over-activity of the PCO. In this sense, NeoSTX has been frequently used in veterinary and human clinics as a long lasting local anesthetic that has many advantages over traditional local anesthetic (Rathmell et al. 2015). Although Nav1.7 has an IC50 100 times higher than that for Nav1.6, the administrated dose of NeoSTX is sufficient to inhibit both channels in nerve terminals of the SON nerve fibers, NeoSTX acts specifically on Nav1.6, which is most highly expressed in peripheral neurons, Ranvier nodules, and dendrites (Caldwell et al. 2000). This channel has an IC50 of $0.86 \mathrm{nM}$. 
In addition, Nav 1.7 is highly expressed in peripheral neurons with a IC50 of $81.6 \mathrm{nM}$ for NeoSTX (Alonso et al. 2016). It is also interesting that the ovary has been described Nav-endocrine sensitive to tetrodotoxin (which has the same molecular target as NeoSTX) in ovarian granulosa cells. This suggests a role in the stimulation of luteolisis and thus tetrodotoxin preserves the luteal phenotype (Bulling et al. 2000). Thus, NeoSTX can regulate sympathetic activity and extend the half-life of the corpora lutea.

Our in vivo studies with NeoSTX were designed to study the role of the toxin to prevent development of the PCO phenotype or to study the capacity of the toxin to reverse the PCO phenotype at the first steps of cyst formation (Lara et al. 2000). To prevent PCO development, we applied the NeoSTX at the same time as EV. Blocking noradrenergic neurons by NeoSTX prevented development of the PCO phenotype, which is shown as the recovery in corpora lutea, decrease in follicular cyst, the increase in plasma levels of progesterone, and decrease in testosterone. All of these factors represent the recovery of estrous cycling activity and probably ovulatory function (Barria et al. 1993). The advantage of using the toxin, especially the local intraovarian delivery, was the method's low invasiveness and reversible. Studies with pulses of the toxin to determine the effective time of action when it was locally administered to the ovary will strengthen the effectiveness of the method. We demonstrated that the NeoSTX decreased norepinephrine in the control rats, and it was also able to decrease norepinephrine in the EV-treated ovaries. It is interesting to show that when NeoSTX was administered 30 days after the $\mathrm{EV}$ administration, i.e. when the ovaries also present the previous steps of follicular cyst formation (Lara et al. 2000), rats quickly recovered their estrous cycling activity, indicating that blocking the neurons could be effective. However, some diffusion of the toxin from the ovary into general circulation may have occurred, and thus, had an unspecific effect on the endocrine system. Our calculation of the toxin dose took into account that the dilution in plasma would result in a concentration that was far from an effective dose. Preventing the development was more effective than the reversion effect. This can be explained because after 30 days of $\mathrm{EV}$ administration, follicular cyst formation began before the toxin was applied, and thus, a new cohort of follicles will be blocked. However, the reversion is consistent with the effect on steroid secretion. We cannot discard a putative effect of the toxin on the nerve terminals from the plexus nerve that could participate in the effect on steroid secretion (Uchida \& Kagitani 2014).
Altogether, these results strongly suggest that NeoSTX could be an innovative drug to block the PCO phenotype, permitting the rats to ovulate and probably recover from decreased fertilization capacity that occurs after a sympathetic discharge, such as chronic sympathetic stress (Riquelme et al.2019), and thus, providing support for the use of this drug as a therapeutic agent for PCOS.

\section{Participation in the study}

MdC participated in the experimental part of the study, biochemical determination data collection, analysis of data and manuscript preparation. NL was in charge of the preparing the toxin and testing the biological activity and purification. HEL conceived the idea and equally contributed to the study design, data collection, analysis, and manuscript preparation.

\section{Declaration of interest}

The authors declare that there is no conflict of interest that could be perceived as prejudicing the impartiality of the research reported.

\section{Funding}

This study was supported by grant Fondecyt 1170291 (to H E L).

\section{References}

Alonso E, Alfonso A, Vieytes MR \& Botana LM 2016 Evaluation of toxicity equivalent factors of paralytic shellfish poisoning toxins in seven human sodium channels types by an automated high throughput electrophysiology system. Archives of Toxicology 90 479-488. (https:// doi.org/10.1007/s00204-014-1444-y)

Banaszewska B, Pawelczyk L \& Spaczynski R 2019 Current and future aspects of several adjunctive treatment strategies in polycystic ovary syndrome. Reproductive Biology 19 309-315. (https://doi.org/10.1016/j. repbio.2019.09.006)

Barria A, Leyton V, Ojeda SR \& Lara HE 1993 Ovarian steroidal response to gonadotropins and beta-adrenergic stimulation is enhanced in polycystic ovary syndrome: role of sympathetic innervation. Endocrinology 133 2696-2703. (https://doi.org/10.1210/ endo.133.6.8243293)

Birmingham K, Gradinaru V, Anikeeva P, Grill WM, Pikov V, Mclaughlin B, Pasricha P, Weber D, Ludwig K \& Famm K 2014 Bioelectronic medicines: a research roadmap. Nature Reviews: Drug Discovery 13 399-400. (https://doi.org/10.1038/nrd4351)

Bulling A, Berg FD, Berg U, Duffy DM, Stouffer RL, Ojeda SR, Gratzl M \& Mayerhofer A 2000 Identification of an ovarian voltageactivated Na+-channel type: hints to involvement in luteolysis. Molecular Endocrinology 14 1064-1074. (https://doi.org/10.1210/ mend.14.7.0481)

Burden HW, Lawrence Jr IE \& Louis T 1985 The adrenergic innervation of the guinea pig ovary during prenatal and postnatal periods. Cells, Tissues, Organs 122 193-196.

Caldwell JH, Schaller KL, Lasher RS, Peles E \& Levinson SR 2000 Sodium channel $\mathrm{Na}(\mathrm{v}) 1.6$ is localized at nodes of ranvier, dendrites, 
and synapses. PNAS 97 5616-5620. (https://doi.org/10.1073/ pnas.090034797)

Chavan SS \& Tracey KJ 2017 Essential neuroscience in immunology. Journal of Immunology 198 3389-3397. (https://doi.org/10.4049/ jimmunol.1601613

Chen H \& Lim CED 2019 The efficacy of using acupuncture in managing polycystic ovarian syndrome. Current Opinion in Obstetrics and Gynecology 31 428-432. (https://doi.org/10.1097/ GCO.0000000000000582)

Cruz G, Barra R, Gonzalez D, Sotomayor-Zarate R \& Lara HE 2012 Temporal window in which exposure to estradiol permanently modifies ovarian function causing polycystic ovary morphology in rats. Fertility and Sterility 98 1283-1290. (https://doi.org/10.1016/j. fertnstert.2012.07.1060)

Curry TE, Lawrence IE \& Burden HW 1985 Ovarian sympathectomy in the golden hamster: effects on estrous cyclicity and follicular development. Experimental and Clinical Endocrinology 86 284-290. (https://doi.org/10.1055/s-0029-1210498)

Del Campo M, Piquer B, Witherington J, Sridhar A \& Lara HE 2019 Effect of superior ovarian nerve and plexus nerve sympathetic denervation on ovarian-derived infertility provoked by estradiol exposure to rats. Frontiers in Physiology 10 349. (https://doi.org/10.3389/ fphys.2019.00349)

Dissen GA, Hill DF, Costa ME, Les Dees CW, Lara HE \& Ojeda SR 1996 A role for trkA nerve growth factor receptors in mammalian ovulation. Endocrinology 137 198-209. (https://doi.org/10.1210/ endo.137.1.8536613)

Dorfman M, Arancibia S, Fiedler JL \& Lara HE 2003 Chronic intermittent cold stress activates ovarian sympathetic nerves and modifies ovarian follicular development in the rat. Biology of Reproduction $\mathbf{6 8}$ 2038-2043. (https://doi.org/10.1095/biolreprod.102.008318)

Flores A, Velasco J, Gallegos AI, Mendoza FD, Everardo PM, Cruz ME \& Dominguez R 2011 Acute effects of unilateral sectioning the superior ovarian nerve of rats with unilateral ovariectomy on ovarian hormones (progesterone, testosterone and estradiol) levels vary during the estrous cycle. Reproductive Biology and Endocrinology 934. (https://doi.org/10.1186/1477-7827-9-34)

Garcia C, Perez F, Contreras C, Figueroa D, Barriga A, Lopez-Rivera A, Araneda OF \& Contreras HR 2015 Saxitoxins and okadaic acid group: accumulation and distribution in invertebrate marine vectors from Southern Chile. Food Additives and Contaminants: Part A, Chemistry, Analysis, Control, Exposure and Risk Assessment 32 984-1002. (https:// doi.org/10.1080/19440049.2015.1028107)

Greiner M, Paredes A, Araya V \& Lara HE 2005 Role of stress and sympathetic innervation in the development of polycystic ovary syndrome. Endocrine 28 319-324. (https://doi.org/10.1385/ ENDO:28:3:319)

Grisk O 2020 The sympathetic nervous system in acute kidney injury. Acta Physiologica 228 e13404. (https://doi.org/10.1111/apha.13404)

Jacobowitz D \& Wallach EE 1967 Histochemical and chemical studies of the autonomic innervation of the ovary. Endocrinology 81 1132-1139. (https://doi.org/10.1210/endo-81-5-1132)

Kuang HY, Li Y, Zhang HP, Legro RS \& Stener-Victorin E 2018 Data do not support effectiveness of acupuncture for improving live birth rate in women with polycystic ovary syndrome. Chinese Journal of Integrative Medicine 24 399-400. (https://doi.org/10.1007/s11655-018-2842-4)

Lansdown A \& Rees DA 2012 The sympathetic nervous system in polycystic ovary syndrome: a novel therapeutic target? Clinical Endocrinology 77 791-801. (https://doi.org/10.1111/cen.12003)

Lara H, Galleguillos X, Arrau J \& Belmar J 1985 Effect of castration and testosterone on norepinephrine storage and on the release of $[(3) \mathrm{H}]$ norepinephrine from rat vas deferens. Neurochemistry International 7 667-674. (https://doi.org/10.1016/0197-0186(85)90064-6)

Lara HE, Mcdonald JK, Ahmed CE \& Ojeda SR 1990a Guanethidinemediated destruction of ovarian sympathetic nerves disrupts ovarian development and function in rats. Endocrinology 127 2199-2209. (https://doi.org/10.1210/endo-127-5-2199)

Lara HE, Mcdonald JK \& Ojeda SR $1990 b$ Involvement of nerve growth factor in female sexual development. Endocrinology 126 364-375. (https://doi.org/10.1210/endo-126-1-364)

Lara HE, Ferruz JL, Luza S, Bustamante DA, Borges Y \& Ojeda SR 1993 Activation of ovarian sympathetic nerves in polycystic ovary syndrome. Endocrinology 133 2690-2695. (https://doi.org/10.1210/ endo.133.6.7902268)

Lara HE, Dissen GA, Leyton V, Paredes A, Fuenzalida H, Fiedler JL \& Ojeda SR 2000 An increased intraovarian synthesis of nerve growth factor and its low affinity receptor is a principal component of steroid-induced polycystic ovary in the rat. Endocrinology 141 1059-1072. (https://doi.org/10.1210/endo.141.3.7395)

Lara HE, Dorfman M, Venegas M, Luza SM, Luna SL, Mayerhofer A, Guimaraes MA, Rosa E Silva AA \& Ramirez VD 2002 Changes in sympathetic nerve activity of the mammalian ovary during a normal estrous cycle and in polycystic ovary syndrome: studies on norepinephrine release. Microscopy Research and Technique 59 495-502. (https://doi.org/10.1002/jemt.10229)

Lawrence IE \& Burden HW 1980 The origin of the extrinsic adrenergic innervation to the rat ovary. Anatomical Record 196 51-59. (https:// doi.org/10.1002/ar.1091960106)

Lim CED, Ng RWC, Cheng NCL, Zhang GS \& Chen H 2019 Acupuncture for polycystic ovarian syndrome. Cochrane Database of Systematic Reviews 7 CD007689. (https://doi.org/10.1002/14651858.CD007689. pub4)

Llewellyn LE 2006 Saxitoxin, a toxic marine natural product that targets a multitude of receptors. Natural Product Reports 23 200-222. (https:// doi.org/10.1039/b501296c)

Lobo K, Donado C, Cornelissen L, Kim J, Ortiz R, Peake RW, Kellogg M, Alexander ME, Zurakowski D, Kurgansky KE, et al. 2015 A Phase 1, dose-escalation, double-blind, block-randomized, controlled trial of safety and efficacy of neosaxitoxin alone and in combination with $0.2 \%$ bupivacaine, with and without epinephrine, for cutaneous anesthesia. Anesthesiology 123 873-885. (https://doi.org/10.1097/ ALN.0000000000000831)

Luna SL, Neuman S, Aguilera J, Brown DI \& Lara HE 2012 In vivo betaadrenergic blockade by propranolol prevents isoproterenol-induced polycystic ovary in adult rats. Hormone and Metabolic Research $\mathbf{4 4}$ 676-681. (https://doi.org/10.1055/s-0031-1301304)

Manriquez V, Castro Caperan D, Guzman R, Naser M, Iglesia V \& Lagos N 2015 First evidence of neosaxitoxin as a long-acting pain blocker in bladder pain syndrome. International Urogynecology Journal 26 853-858. (https://doi.org/10.1007/s00192-014-2608-2)

Marcondes FK, Bianchi FJ \& Tanno AP 2002 Determination of the estrous cycle phases of rats: some helpful considerations. Brazilian Journal of Biology 62 609-614. (https://doi.org/10.1590/s151969842002000400008)

Neilson D, Jones GS, Woodruff JD \& Goldberg B 1970 The innervation of the ovary. Obstetrical and Gynecological Survey 25 889-904. (https:// doi.org/10.1097/00006254-197010000-00001)

Pastelin CF, Rosas NH, Morales-Ledesma L, Linares R, Dominguez R \& Moran C 2017 Anatomical organization and neural pathways of the ovarian plexus nerve in rats. Journal of Ovarian Research 1018. (https://doi.org/10.1186/s13048-017-0311-x)

Pavlov VA \& Tracey KJ 2017 Neural regulation of immunity: molecular mechanisms and clinical translation. Nature Neuroscience 20 156-166. (https://doi.org/10.1038/nn.4477)

Pikov V, Sridhar A \& Lara HE 2018 High-frequency electrical modulation of the superior ovarian nerve as a treatment of polycystic ovary syndrome in the rat. Frontiers in Physiology 9 459. (https://doi. org/10.3389/fphys.2018.00459)

Rathmell JP, Strichartz G \& Wanderer J 2015 Neosaxitoxin versus traditional local anesthetics: mechanism of action and sites of https://joe.bioscientifica.com https://doi.org/10.1530/JOE-19-0545 (c) 2020 Society for Endocrinology Published by Bioscientifica Ltd. Printed in Great Britain 
notable effect. Anesthesiology 123 A23. (https://doi.org/10.1097/01. anes.0000470956.02665.a6)

Riquelme G, Sepulveda JM, Al Ghumgham Z, Del Campo M, Montero C \& Lagos N 2018 Neosaxitoxin, a paralytic shellfish poison toxin, effectively manages bucked shins pain, as a local long-acting pain blocker in an equine model. Toxicon 141 15-17. (https://doi. org/10.1016/j.toxicon.2017.11.004)

Riquelme R, Ruz F, Mayerhofer A \& Lara HE 2019 Role of ovarian sympathetic nerves and cholinergic local system during cold stress. Journal of Endocrinology 242 115-124. (https://doi.org/10.1530/JOE19-0125)

Rodriguez-Navarro AJ, Lagos N, Lagos M, Braghetto I, Csendes A, Hamilton J, Figueroa C, Truan D, Garcia C, Rojas A, et al. 2007a Neosaxitoxin as a local anesthetic: preliminary observations from a first human trial. Anesthesiology 106 339-345. (https://doi. org/10.1097/00000542-200702000-00023)

Rodriguez-Navarro AJ, Saenz R \& Navarrete C 2007b Esophageal stenosis in epidermolysis bullosa. Gastrointestinal Endoscopy 66 1067. (https:// doi.org/10.1016/j.gie.2007.05.003)

Rosas G, Linares R, Ramirez DA, Vieyra E, Trujillo A, Dominguez R \& Morales-Ledesma L 2018 The neural signals of the superior ovarian nerve modulate in an asymmetric way the ovarian steroidogenic response to the vasoactive intestinal peptide. Frontiers in Physiology 9 1142. (https://doi.org/10.3389/fphys.2018.01142)

Stener-Victorin E, Jedel E \& Manneras L 2008 Acupuncture in polycystic ovary syndrome: current experimental and clinical evidence. Journal of Neuroendocrinology 20 290-298. (https://doi.org/10.1111/j.13652826.2007.01634.x)

Stener-Victorin E, Jedel E, Janson PO \& Sverrisdottir YB 2009 Lowfrequency electroacupuncture and physical exercise decrease high muscle sympathetic nerve activity in polycystic ovary syndrome. American Journal of Physiology: Regulatory, Integrative and Comparative Physiology 297 R387-R395. (https://doi.org/10.1152/ ajpregu.00197.2009)

Toufexis D, Rivarola MA, Lara H \& Viau V 2014 Stress and the reproductive axis. Journal of Neuroendocrinology 26 573-586. (https:// doi.org/10.1111/jne.12179)
Uchida S \& Kagitani F 2014 Effects of electrical stimulation of autonomic nerves to the ovary on the ovarian testosterone secretion rate in rats. Autonomic Neuroscience: Basic and Clinical 180 48-52. (https://doi. org/10.1016/j.autneu.2013.10.007)

Urra J, Blohberger J, Tiszavari M, Mayerhofer A \& Lara HE 2016 In vivo blockade of acetylcholinesterase increases intraovarian acetylcholine and enhances follicular development and fertility in the rat. Scientific Reports 6 30129. (https://doi.org/10.1038/srep30129)

Velasquez M, Fernandez L, Leib CS, Orozco V, Pava F \& Perafan P 2019 Cardiac sympathetic denervation for conventional treatment refractory arrhythmias. Archivos de Cardiología de México 89 211-215. (https://doi.org/10.24875/ACM.M19000052)

Venegas B, De Leon Gordillo LY, Rosas G, Espinoza JA, Moran C, Dominguez R \& Morales-Ledesma L 2019 In rats with estradiol valerate-induced polycystic ovary syndrome, the acute blockade of ovarian beta-adrenoreceptors improve ovulation. Reproductive Biology and Endocrinology 17 95. (https://doi.org/10.1186/s12958-019-0539-y)

Wakade AR 1979 Recycling of noradrenergic storage vesicles of isolated rat vas deferens. Nature 281 374-376. (https://doi.org/10.1038/281374a0)

Wakade AR 1980 A maximum contraction and substantial quantities of tritium can be obtained from tetraethylammonium-treated $[3 \mathrm{H}]$-noradrenaline preloaded, rat vas deferens in response to a single electrical shock. British Journal of Pharmacology 68 425-436. (https:// doi.org/10.1111/j.1476-5381.1980.tb14556.x)

Zamorano R, Marin M, Cabrera F, Figueroa D, Contreras C, Barriga A, Lagos N \& Garcia C 2013 Determination of the variability of both hydrophilic and lipophilic toxins in endemic wild bivalves and carnivorous gastropods from the southern part of Chile. Food Additives and Contaminants: Part A, Chemistry, Analysis, Control, Exposure and Risk Assessment 30 1660-1677. (https://doi.org/10.1080/19440049.20 13.805438)

Zar JH 1984 Biostatistical Analysis. Englewood Cliffs, NJ: Prentice-HaIl.

Zheng Y, Stener-Victorin E, Ng EH, Li J, Wu X \& Ma H 2015 How does acupuncture affect insulin sensitivity in women with polycystic ovary syndrome and insulin resistance? Study protocol of a prospective pilot study. BMJ Open 5 e007757. (https://doi.org/10.1136/ bmjopen-2015-007757)

Received in final form 5 January 2020

Accepted 16 January 2020

Accepted Manuscript published online 20 January 2020 (c) 2020 Society for Endocrinology Published by Bioscientifica Ltd. Printed in Great Britain 